\title{
Discussion Table Ronde GECO sur les entorses fraîches externes de cheville
}

\author{
P. Grumillier \\ Clinique de la Providence, 14, 16, place Ch. de Gaulle, F-55200 Commercy
}

Plusieurs intervenants ont pris la parole au cours de cette discussion. Tout d'abord Philippe Segal (Reims), se dit particulièrement satisfait de l'évolution des idées tendant à diminuer les indications chirurgicales, lui même n'opérant pratiquement plus les entorses fraîches graves de cheville.

Le Professeur Duquennoy (Lille) apporte deux remarques:

1) Il reste partisan dans certains cas d'un traitement chirurgical en précisant que ce traitement doit rester aussi peu aggressif que possible et doit à l'extrême se contenter de l'évacuation des caillots qui peuvent être responsable d'une mauvaise cicatrisation des lésions ligamentaires.

2) En présentant une étude statistique scandinave récente, il confirme la tendance à la diminution des indications chirurgicales. Il semble, d'après cette étude et les commentaires du Professeur D. que si l'on veut obtenir un résultat rapide, il vaille mieux opter pour le traitement fonctionnel alors que si l'on veut être sûr d'éviter une laxité résiduelle, il faille choisir la chirurgie.

Les chirurgiens de la Clinique $d u$ Parc de Lyon font part de leur expérience considérable (800 cas par an depuis 13 ans) et confirment qu'ils ne réalisent plus aucun geste chirurgical sur les entorses graves de cheville sans constater de laxité secondaire.

Le Docteur Jean Yves Jenny (Stras- bourg) rapporte d'autres études récentes qui vont dans le même sens en montrant que le traitement chirurgical n'a pratiquement plus d'avantage dans les études randomisées et prospectives.

Mesnel du Mans apporte l'exemple d'une entorse grave de l'enfant avec un gros fragment du péronier. Il s'agit probablement d'un arrachement ligamentaire et il confirme la nécessité d'un cliché en varus forcé chez l'enfant afin de faire apparaître le gros fragment et d'éviter une erreur de diagnostic.

Le Docteur Jean Berger (Strasbourg) est d'accord avec cette attitude et mentionne à nouveau, le problème du diagnostic différentiel avec la spasticité.

Le Docteur Jean Claude Dosch (Strasbourg) pose deux questions : le testing est-il réalisé par le Docteur Max Perrin sous $\mathrm{A} / \mathrm{G}$ ? et le chiffre de $15^{\circ}$ de différence dans les clichés comparatifs lui semble modeste pour le diagnostic d'entorse grave chez les chirurgiens de Colmar.

Le Docteur Max Perrin (Dijon) répond que les clichés n'ont jamais été pratiqués sous anesthésie générale et que dans les quelques cas où une $\mathrm{A} / \mathrm{G}$ a été réalisée, les conclusions ont souvent été excessives avec des diagnostics érronés d'entorses graves.

Réponse du Docteur Balliet (Colmar) : les études réalisées ont toujours été précédées d'élimination des patients présentant une laxité constitutionnelle.
Le chiffre moyen observé des entorses graves de différence de varus comparatif est de $18^{\circ}$

Le Docteur Demigneux (Troyes) considère que le cliché en varus doit être réalisé sous $\mathrm{A} / \mathrm{G}$. Il pense que le cliché en tiroir antérieur est particulièrement intéressant à réaliser et souvent moins douloureux. Il pose la question de savoir quelle est la conduite à tenir devant une lésion du ligament péronéo tibial inférieur.

Le Docteur Grumillier (Commercy) précise que, dans l'étude du GECO, aucune lésion du ligament péronéo tibial inférieur n'a été notée dans les compte rendus opératoires. La capsule articulaire étant souvent déchirée mais en dessous du niveau de l'articulation péronéo tibiale.

Enfin la question est posée par plusieurs intervenants afin de connaître la nécessité d'interdire l'appui précoce dans le cas des entorses graves de cheville traitées orthopédiquement. La réponse du Docteur Grumillier et du Docteur Balliet est la suivante : la mise en décharge de la cheville pendant les 2 ou 3 premières semaines permet la résorption de l'hémarthrose fréquente de la sous astragalienne et la mise en place secondaire de l'attelle pneumatique est facilitée après disparition de l'oedème qui est accélérée par la mise en décharge et en surélévation pendant quelques jours de la cheville. 